\title{
An Ultrasonographic Multiparametric Carotid Plaque Risk Index Associated with Cerebrovascular Symptomatology: A Study Comparing Color Doppler Imaging and Contrast-Enhanced Ultrasonography
}

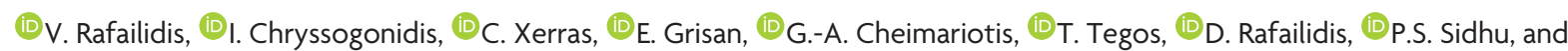
(D) A. Charitanti-Kouridou

\begin{abstract}
BACKGROUND AND PURPOSE: Various ultrasonographic features of carortid plaques have been associated with the occurence of stroke, highlighting the need for multi-parametric assessment of plaque's vulnerability. Our aim was to compare ultrasonographic multiparametric indices using color Doppler imaging and contrast-enhanced sonography between symptomatic and asymptomatic carotid plaques.
\end{abstract}

MATERIALS AND METHODS: This was a cross-sectional observational study recruiting 54 patients (72.2\% male; median age, 61 years) undergoing sonography and contrast-enhanced sonography. Patients were included if a moderately or severely stenotic internal carotid artery plaque was detected, with the plaque being considered symptomatic if it was ipsilateral to a stroke occuring within the last 6 months. A vulnerability index, previously described by Kanber et al, combined the degree of stenosis, gray-scale median, and a quantitative measure of surface irregularities (surface irregularity index) derived from color Doppler imaging and contrast-enhanced ultrasonography, resulting in 2 vulnerability indices, depending on the surface irregularity index used. Mann-Whitney $U$ and $t$ tests were used to compare variables between groups, and receiver operating characteristic curves were used to compare diagnostic accuracy.

RESULTS: Sixty-two plaques were analyzed (50\% symptomatic), with a mean degree of stenosis of $68.9 \%$. Symptomatic plaques had a significantly higher degree of stenosis (mean, 74.7\% versus 63.1\%; $P<.001$ ), a lower gray-scale median (13 versus 38; $P=.001$ ), and a higher Kanber vulnerability index based both on color Doppler imaging (median, 61.4 versus $16.5 ; P<.001$ ) and contrast-enhanced ultrasonography (median, 88.6 versus $25.2 ; P<.001$ ). The area under the curve for the detection of symptomatic plaques was 0.772 for the degree of stenosis alone, 0.783 for the vulnerability index-color Doppler imaging, and 0.802 for the vulnerability index-contrast-enhanced ultrasonography, though no statistical significance was achieved.

CONCLUSIONS: Symptomatic plaques had a higher degree of stenosis, lower gray-scale median values, and higher values of the Kanber vulnerability index using both color Doppler imaging and contrast-enhanced ultrasonography for plaque surface delineation.

ABBREVIATIONS: $\mathrm{AUC}=$ area under the curve; $\mathrm{CEUS}=$ contrast-enhanced ultrasonography; $\mathrm{CDI}=$ color Doppler imaging; $\mathrm{DOS}=$ degree of stenosis; $\mathrm{GSM}=$ gray-scale median; IQR = interquartile range; $\mathrm{ROC}=$ receiver operating characteristic; $\mathrm{SII}=$ surface irregularity index; $\mathrm{US}=$ ultrasonography; $\mathrm{VI}=\mathrm{vulnerability}$ index

C arotid atherosclerosis accounts for approximately $10 \%-15 \%$ of all strokes, with thromboembolism from a moderate or severe stenosis representing the underlying mechanism. The degree of internal carotid artery stenosis has long been used as the

Received November 12, 2018; accepted after revision March 30, 2019.

From the Department of Radiology (V.R., I.C., A.C.-K.) and First Department of Neurology (C.X., T.T.), AHEPA University General Hospital, Aristotle University of Thessaloniki, Thessaloniki, Greece; Department of Information Engineering (E.G.), University of Padova, Padova, Italy; School of Imaging Sciences and Biomedical Engineering (E.G.), King's College London, London, UK; Laboratory of Computing (G.-A.C.), Medical Informatics and Biomedical-Imaging Technologies, School of Medicine, Aristotle University of Thessaloniki, Thessaloniki, Greece; Department of Radiology (D.R.), "G. Gennimatas" General Hospital of Thessaloniki, Thessaloniki, Greece; and Department of Radiology (P.S.S.), King's College Hospital, London, UK. V.R. received a scholarship for his PhD studies on "Imaging of the Carotid Vulnerable Plaque with Contrast-Enhanced Ultrasound and Multi-Detector Computed Tomography Angiography" from the Alexander S. Onassis Public Benefit Foundation (grant No. G ZJ 050-2/2015--2016). primary parameter considered for guiding treatment of patients with carotid disease. Nevertheless, it is now well-established that additional plaque features contribute to the vulnerability of the plaque, a term corresponding to the potential of the plaque for stroke or transient ischemic attack. Such features include the composition and surface morphology of the plaque and can be investigated using any imaging technique, from conventional ultrasonography (US) to noninvasive cross-sectional imaging modalities such as multidetector CT angiography. ${ }^{1-5}$ Ultrasonography is valuable for the diagnosis of carotid disease and is the

Please address correspondence to Vasileios Rafailidis, MD, MSc, PhD, Department of Radiology, AHEPA University General Hospital, Aristotle University of Thessaloniki, Thessaloniki, Greece, St. Kiriakidis 1, P.C. 54636, Thessaloniki, Greece (Hellas); e-mail: billraf@hotmail.com

$\triangle \Delta$ Indicates article with supplemental on-line photos.

http://dx.doi.org/10.3174/ajnr.A6056 
first-line technique with inherent advantages including low cost, repeatability, and high accuracy. ${ }^{6}$ Plaque composition can be ultrasonographically evaluated both subjectively and quantitatively using the gray-scale median (GSM) technique, already proved to correlate with neurovascular symptoms and representing a risk factor for stroke. ${ }^{7-13}$

Plaque surface morphology, either in the form of mere irregularities or true ulceration, has also been associated with the occurrence of neurovascular symptoms. Consequently, surface characterization should be an essential part of any imaging examination. Plaque surface can be subjectively classified as smooth, irregular, or ulcerated, but quantitative measures would be more beneficial because they are less operator-dependent and more objective. ${ }^{2,3,14}$ There has been an effort to introduce a quantitative measure of surface irregularities, providing some initial promising results. ${ }^{15,16}$ In light of these results, it is evident that the introduction and use of a mathematic index combining features of plaque vulnerability are now feasible. Initial reports have already been published by Kanber et al, ${ }^{17}$ with encouraging results, indicating that multiparametric indices outperform the degree of stenosis (DOS) alone for the detection of symptomatic plaques. The use of intravenously administered microbubbles acting as ultrasonographic contrast agents has been introduced, and the technique of contrast-enhanced ultrasonography (CEUS) has been investigated in the assessment of carotid disease. CEUS has been shown to improve carotid plaque delineation compared with the color Doppler imaging (CDI) technique, being independent of artifacts such as overwriting, aliasing, and Doppler angle dependency, and could be a valuable alternative to CDI for quantification of surface irregularities. ${ }^{14,18-20}$

The purpose of this study was to investigate the value of a multiparametric ultrasonographic carotid plaque risk index, previously described by Kanber et al, ${ }^{17}$ in detecting symptomatic plaques by comparing values between symptomatic and asymptomatic plaques and comparing its diagnostic accuracy with the well-established parameter of DOS. The index investigated is the carotid plaque risk index introduced by Kanber et al, incorporating stenosis, GSM, and a quantitative measure of surface irregularities estimated on the basis of both CDI and CEUS images, known to provide optimal carotid plaque surface delineation. The index will be referred to as the Kanber vulnerability index (VI).

\section{MATERIALS AND METHODS}

\section{Patients and Inclusion/Exclusion Criteria}

The institutional ethics review board (Committee of Bioethics and Deontology, School of Medicine, Aristotle University of Thessaloniki) approved this study, and every patient recruited provided written informed consent. Recruitment of patients was performed in a prospective and consecutive pattern from the Radiology and Neurology Department from April 2016 to April 2018 (2 years). Both symptomatic and asymptomatic patients were recruited after being referred for ultrasonography either due to the occurrence of stroke or for unrelated or screening purposes. A patient was considered symptomatic if a stroke had occurred within the past 6-month period, based on the patient's history, clinical examination, or review of radiologic studies. Moreover, an internal carotid artery plaque with moderate $(50 \%-69 \%)$ or severe (70\%-99\%) stenosis should have been documented on the US examination based on previously reported velocity criteria. ${ }^{21}$ A $<50 \%$ plaque was only included in the study if it was located contralateral to a $\geq 50 \%$ plaque. Both carotid systems were analyzed separately in every patient studied. Plaques situated ipsilateral to the stroke were considered symptomatic, while those situated contralateral to the stroke were considered asymptomatic.

Exclusion criteria included a history of allergy or other contraindication to the US contrast agents used, the presence of neurologic diseases mimicking stroke, and a clinical presentation and history of comorbidities that could cause stroke (such as arrhythmias, cardiac anatomic abnormalities, thrombophilia, and immunologic diseases such as antiphospholipid syndrome). In detail, cardiac anatomic conditions that were excluded as potential sources of embolism included the following: left ventricular aneurysm, intracardiac thrombus or myxoma, left ventricular dysfunction or hypokinesis, a patent foramen ovale, a mechanical prosthetic cardiac valve, and valvular dysfunction such as mitral stenosis. Extensively calcified plaques with acoustic shadowing were also excluded from analysis if considered unsuitable for quantification. Namely, the decision to exclude a plaque was made by the 2 observers analyzing the images on the basis of consensus. A plaque was regarded as extensively calcified and was excluded when situated on the near wall (proximal to the probe), thus causing acoustic shadowing hiding the plaque surface even for a small part, if this shadowing was not possible to overcome by changing the probe position and direction of sonography beam. Conversely, when the plaque was located on the distal wall (distal to the probe) and its surface was visible, it was not excluded. The decision to exclude a plaque was made before quantification analysis.

\section{Imaging Technique}

All patients included in the study underwent US and CEUS in both carotid systems. US examinations were performed by an experienced radiologist (9 years of experience) with a Logiq S8 (GE Healthcare, Milwaukee, Wisconsin) with an XDclear technology device and a linear probe (type 9L) with a 3- to $10-\mathrm{MHz}$ bandwidth. B-mode and CDI were used for the routine part of US, including grading of stenosis using the Society of Radiologists in Sonography Consensus velocity criteria $^{21}$ and diametric measurements on axial CDI and evaluation of plaque characteristics. For accurate grading of stenosis, the diametric measurements on axial CDI, the peak systolic velocities, and the peak systolic velocity ratios were taken into consideration. If a suitable plaque was detected and the patient met the inclusion criteria, a CEUS examination was performed within 1 week following the conventional US. CEUS was performed with the intravenous administration of $2.4 \mathrm{~mL}$ of sulphur hexafluoride microbubbles (SonoVue, Bracco, Milan, Italy) followed by 10 $\mathrm{mL}$ of saline and using the contrast-specific mode of the device. The mechanical index was kept to $<0.1$, and the gain was suitably adjusted to achieve optimal microbubble visualization.

\section{Image Analysis}

Video clips of the affected internal carotid artery in the long axis plane were recorded by the radiologist performing the examination, both on CDI and CEUS. The clips were then reviewed by 2 
different radiologists, observers (A and B) who had 11 years of experience and were specifically trained for this study on the use of the software, blinded to the patient's history. The 2 observers chose a single frame of CDI and the CEUS video clip optimally visualizing the carotid plaque surface. Subsequently, 1 observer used the surface irregularities software developed for this study to quantify surface irregularities, while both observers analyzed the images in the first 30 cases for interobserver agreement analysis, being blinded to each other's results.

For the evaluation of the GSM, previously described techniques were used $^{9,22}$ based on Adobe Photoshop CS6 (Adobe Systems, San Jose, California). In brief, B-mode images in JPEG format were used, with CDI helping delineate hypoechoic plaques. The same presettings were initially used for the acquisition of B-mode images in all patients. The images were normalized before analysis using linear scaling, by setting the value of zero to the echogenicity of blood and the value of 190 to the adventitia. Once the normalization was complete, a closed ROI was drawn over the plaque, and the echogenicity histogram and GSM value were calculated.

The application used for quantification of surface irregularities in this study was developed on the basis of Matlab, Version R2017b (MathWorks, Natick, Massachusetts) and is able to quantify the surface irregularities of the plaque. The physician using this software manually places successive points on the plaque surface and the outer blood vessel wall, for both the upper and lower vessel wall, depending on the distribution of the plaque. As previously described by Kanber et al, ${ }^{16,17}$ a plaque surface irregularity index (SII) is calculated by the computational summation of the angular deviations of the luminal plaque surface from the straight line, divided by the physical length of the plaque surface. The equation used was the following: $(\varphi 1+\varphi 2+\varphi 3+\varphi \nu) / 1$, where $\varphi$ represents the angle of deviation from the straight line, and 1 , the plaque surface length. ${ }^{16,17}$ The cosine rule was used for calculating the surface irregularity index as explained in detail in On-line Fig 1. The surface of the plaque is delineated by color Doppler blood flow signals on the CDI and the border of the microbubble column on CEUS.

Given that the US images used by the software were in an appropriately calibrated JPEG format, pixel spacing and length information were available for the software. In detail, the JPEG images were calibrated on the basis of a scale available in all US images and using a calibration tool developed for this software. As a result, the software can measure the required angles by using the cosine rule for the triangles formed by the consecutive points placed by the physician. The SII of the carotid wall with the greater plaque surface irregularities was recorded for analysis because this was considered more clinically relevant for the detection of symptoms. Only 1 measurement of the SII was performed in each plaque and using the single static image chosen in each case. The Kanber vulnerability index was defined as $(\mathrm{DOS} \times \mathrm{SII}) /(\mathrm{GSM}+1)$ in concordance with a previous study that introduced this index, ${ }^{17}$ and 2 such indices were calculated on the basis of the SII value derived from the CDI or CEUS images (SII-CDI, SII-CEUS, VI-CDI, and VI-CEUS, respectively).

\section{Statistical Analysis}

SPSS, Version 23.0 (IBM, Armonk, New York) was used for statistical analysis. Descriptive statistics included mean and SD for normally distributed variables and median and interquartile range (IQR) for non-normally distributed variables. The Kolmogorov-Smirnov test was used to test the normal distribution of variables. The intraclass correlation coefficient was used to assess interobserver agreement of the SII. Both observers quantified the SII only for the interobserver agreement analysis in a part of the sample, while the more experienced radiologist quantified the SII in all patients for diagnostic accuracy measures. Mann-Whitney $U$ and $t$ tests were used to compare means between groups, depending on the normality of distribution. Receiver operating characteristic (ROC) analysis was used for diagnostic accuracy analysis. Statistical significance level was set at .05. For comparisons between areas under the ROC curves, $P<.017$ was considered statistically significant (Bonferroni correction for 3 pair-wise comparisons). Comparisons between areas under the ROC curve were performed with MedCalc for Windows (MedCalc Software, Mariakerke, Belgium).

\section{RESULTS}

\section{Patients}

Fifty-four patients (39 males) were recruited prospectively, with 14 patients showing bilateral plaques. The median age was 61 years, and the IQR was 17. In total, 62 plaques (31 symptomatic and 31 asymptomatic) met the inclusion criteria and were studied, while 6 plaques were excluded due to extensive calcification with acoustic shadowing hindering quantitative analysis. The remaining carotid systems had no atherosclerotic lesions detected. The mean DOS was $68.9 \% \pm 12.8 \%$. The median GSM was 22.5 $(\mathrm{IQR}=31.5)$. The median SII-CDI was $10.9(\mathrm{IQR}=8.6)$, while the median SII-CEUS was $11.4(\mathrm{IQR}=10.5)$. No adverse reaction to the US contrast agent was observed. Median VI-CDI and VICEUS were 37.3 and 65.2 (IQR $=34.4$ and 91.5), respectively.

\section{SII Interobserver Agreement}

An initial analysis was performed in the first 30 cases to determine the reproducibility and interobserver agreement of the SII by estimating the intraclass correlation coefficient and limits of agreement. The intraclass correlation coefficient for SII-CDI was 0.954 (95\% confidence interval, 0.904-0.978), while for SII-CEUS, it was 0.963 (95\% CI, 0.923-0.983), both measures indicating very good agreement for the SII parameter, with CEUS providing slightly better agreement. The mean, SD, and lower and upper limits of agreement for SII-CDI and SII-CEUS were - 1.1 and 3.3; -7.6 and 5.3 ; -0.4 and 4.3 ; and -9 and 8 , respectively.

\section{Comparison of Parameters between Symptomatic and Asymptomatic Plaques}

The DOS was found to statistically significantly differ between symptomatic and asymptomatic plaques, based on the $t$ test $(P<$ .001). The Mann-Whitney $U$ test confirmed statistical significance in the difference of values between asymptomatic and symptomatic plaques for the GSM $(P=.001)$, VI-CDI $(P<.001)$, VI-CEUS $(P<.001)$, but not for SII-CDI alone $(P=.17)$ and SII-CEUS, for which a trend toward significance was detected $(P=.055)$. The mean and SD values of the previous variables can be found in Table 1. The corresponding boxplots can be found in Fig 1. 


\section{ROC Analysis for the Detection of Symptomatic Plaque}

ROC analysis was performed to compare the diagnostic accuracy of the DOS and the Kanber VI, including the SII deriving from both CDI and CEUS for the detection of symptomatic plaque. The areas under the curve (AUCs) with the respective 95\% CI are presented in Table 2, while the ROC curves are shown in Fig 2. Screening and optimal and diagnostic cutoff values were defined for the DOS, VI-CDI, and VI-CEUS to achieve superior sensitivity, balanced sensitivity and specificity, and superior specificity, respectively. Screening and optimal and diagnostic cutoff values

Table 1: Descriptive statistics and significance of comparisons between ultrasonography parameters of symptomatic and asymptomatic plaques

\begin{tabular}{lccccccc}
\hline & \multicolumn{2}{c}{ Asymptomatic } & & \multicolumn{2}{c}{ Symptomatic } & \\
\cline { 2 - 3 } & Median & IQR & & Median & IQR & & $P$ Value \\
\hline DOS $^{\mathrm{a}}$ & 63.1 & 11.8 & & 74.7 & 11.1 & $<.001^{\mathrm{b}}$ \\
GSM & 38 & 27 & & 13 & 19 & $.001^{\mathrm{b}}$ \\
SII-CDI & 9.5 & 7.5 & & 12 & 9.1 & .17 \\
SII-CEUS & 9.8 & 6.9 & & 12.9 & 11 & .055 \\
VI-CDI & 16.5 & 29.2 & & 61.4 & 109.7 & $<.001^{\mathrm{b}}$ \\
VI-CEUS & 25.2 & 33.3 & & 88.6 & 122.1 & $<.001^{\mathrm{b}}$ \\
\hline
\end{tabular}

${ }^{a}$ Mean and SD are presented for DOS only (normally distributed variable).

${ }^{\mathrm{b}}$ Significant difference.
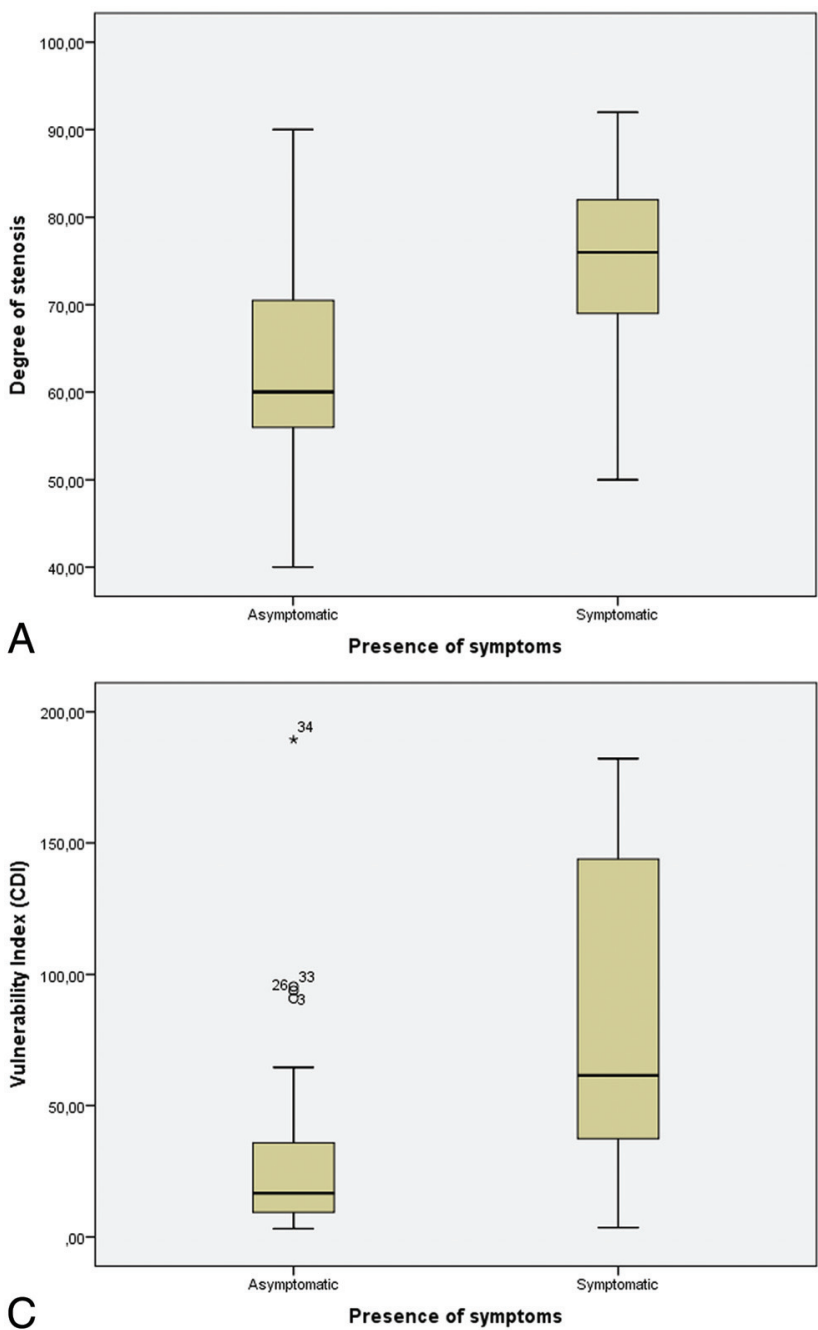

were defined for VI (CDI) as 8.6, 17.3, and 104.3, respectively. The respective resulting sensitivity and specificity for the detection of symptomatic plaques were $96.8 \%$ and $19.4 \%, 87.1 \%$ and $54.8 \%$, and $35.5 \%$ and $96.8 \%$. For VI (CEUS), the cutoff values defined were 7.7 (screening), 29 (optimal), and 134.8 (diagnostic), yielding sensitivity and specificity values of $96.8 \%$ and $16.1 \%, 87.1 \%$ and $71 \%$, and $35.5 \%$ and $96.8 \%$, respectively. In ROC curve analysis, the areas under the curve of the DOS (area under the curve $=$ $0.77 \pm 0.06)$, VI (CDI) (area under the curve $=0.78 \pm 0.06$ ), and VI (CEUS) (area under the curve $=0.80 \pm 0.06$ ) were not statistically significantly different $(P>.017)$. On the basis of these results, both vulnerability indices achieved a higher area under the curve than the DOS alone for the detection of symptomatic plaques, though no statistical significance was achieved. The greatest area under the curve was achieved when the SII-CEUS was used. Examples of quantitative analysis of an asymptomatic and symptomatic plaque can be found in On-line Figs 2 and 3, respectively.

\section{DISCUSSION}

The present study investigated the value of a previously described multiparametric ultrasonographic risk index (the Kanber carotid
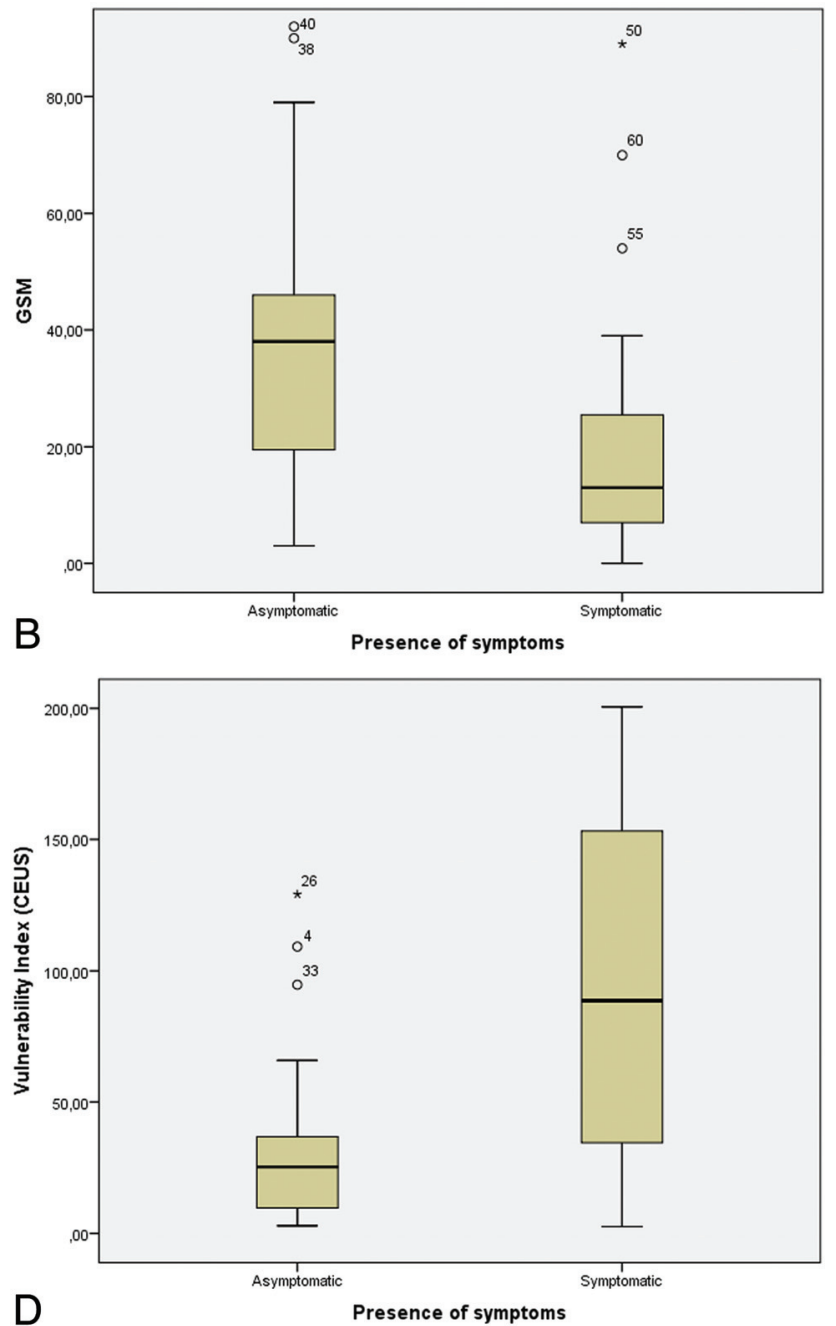

FIG 1. Boxplots comparing the degree of stenosis (A), GSM (B), VI-CDI (C), and VI-CEUS (D) between asymptomatic and symptomatic plaques (62 plaques from 54 patients were included). 
plaque risk index or VI) for the detection of symptomatic carotid plaques. The VI combined the DOS with the GSM and a quantitative measure of surface irregularities, derived both from CDI and CEUS, because the latter is considered to provide optimal plaque delineation. The study results show that the DOS, GSM, and both VIs significantly differed between asymptomatic and symptomatic plaques. On ROC analysis, both multiparametric VIs had higher areas under the curve than the DOS, with the VI using the SII measured on CEUS achieving the greatest area under the curve for the detection of symptomatic plaques. No statistical significance was reached when comparing ROC curves for potential reasons that will be discussed later.

The concept of carotid vulnerable plaque is currently wellestablished, with certain imaging features being regarded as risk factors for the occurrence of neurovascular symptoms. Such features include plaque composition and surface morphology, both adequately studied with US. ${ }^{2,3,14}$ Consequently, the idea of a multiparametric mathematic index incorporating features of vulnerability with the reference variable of the DOS was introduced, and initial effort was made to conceive and evaluate such indices. Prati $\mathrm{el} \mathrm{al}^{23}$ proposed a VI combining stenosis with subjective measures of surface morphology and plaque composition in the form of echogenicity and texture, achieving an AUC of 0.9 , outperforming the accuracy of the Framingham Risk Score alone (0.88) for the prediction of stroke. A later study combined the DOS with an

Table 2: ROC analysis for the detection of symptomatic plaques

\begin{tabular}{lccc} 
& & \multicolumn{2}{c}{$95 \% \mathrm{CI}$} \\
\cline { 3 - 4 } Test Examined & AUC & Lower & Upper \\
\hline DOS & 0.772 & 0.653 & 0.89 \\
VI (CDI) & 0.783 & 0.666 & 0.899 \\
VI (CEUS) & 0.802 & 0.689 & 0.916 \\
\hline
\end{tabular}

\section{ROC Curve}

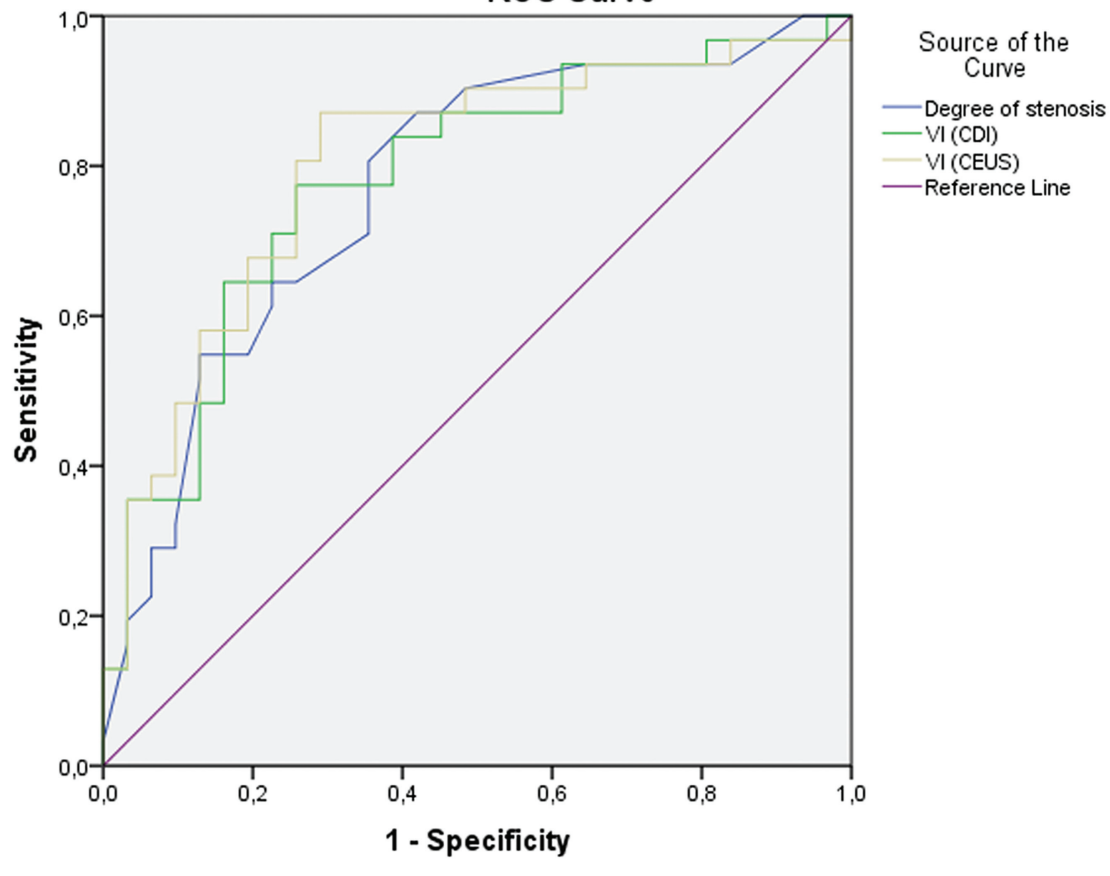

Diagonal segments are produced by ties. objective arithmetic variable describing the echogenicity of plaque, concluding that the resulting index was significantly higher in symptomatic patients and had greater prognostic value compared with stenosis or echogenicity alone. This study also determined a cutoff value of 0.36 for optimal discrimination of symptomatic and asymptomatic patients. ${ }^{24}$

In another type of index, termed the "activity index," the DOS has been combined with the GSM and subjective measures of echogenicity heterogeneity, juxtaluminal echolucent area, and plaque surface interruption. This index was significantly higher in symptomatic plaques, with a cutoff point of 52 being determined for optimal discrimination. ${ }^{25}$ The computation of such an index using ultrasonographic images was also made possible with the development of a stand-alone computer-assisted diagnosis software, allowing the physician to readily assess plaque vulnerability in everyday clinical practice. ${ }^{26}$ Nicolaides et $\mathrm{al}^{27}$ have investigated the value of 3 risk-stratification models in a prospective multicenter study of patients with moderate or severe stenosis, concluding that the DOS alone has an AUC of 0.59, while if combined with clinical features or clinical and ultrasonographic features of vulnerability, the AUC is increased to 0.66 and 0.82 , respectively. Both the GSM and discrete white areas proved to be independent predictors of ipsilateral cerebral or retinal ischemia. ${ }^{27}$

Despite these promising initial results, a disadvantage of these studies lies in the use of subjective variables, which are limited by lower interobserver agreement and reproducibility and increased operator dependency. The study by Kanber et $\mathrm{al}^{17}$ suggested a mathematic index exclusively using quantitative variables for DOS, GSM, and surface irregularity. This index was used in the current study, but using different software and manual delineation of the plaque surface and applying both CDI and CEUS techniques for improved plaque surface delineation. This study concluded that the risk index is significantly higher in symptomatic plaques, also being superior in terms of diagnostic accuracy for the detection of symptomatic plaques compared with stenosis alone. The GSM and SII combined with stenosis achieved an AUC of 0.849 , outperforming stenosis alone (AUC $=0.771$ ). In keeping with these results, the present study calculated an AUC of 0.772 for stenosis alone, 0.783 for VI-CDI, and 0.802 for VI-CEUS. ${ }^{17}$ In our study, the median values of the index in symptomatic (asymptomatic) plaques were 16.5 (61.47) for the CDI technique and 25.2 (88.66) for CEUS, highlighting a greater difference in value in the latter case. These findings confirm the well-established superiority of CEUS to CDI for the delineation of carotid plaque surface free of artifacts and indicate that the CEUS technique provides more clinically significant information regarding surface irregularities. ${ }^{18-20,28-30}$ Multiparametric analysis of carotid plaques can

FIG 2. ROC curves for the detection of symptomatic plaques by the compared variables. 
also be performed through integrated classification systems like the PLAC-RISK score, combining clinical data, the DOS, echogenicity, surface characteristics, and intraplaque neovascularization, to calculate a score with the purpose of suggesting optimal patient management. ${ }^{31}$

The current study has some limitations, including a relatively small number of patients, a potential spectrum bias because only patients with at least 1 plaque with moderate or severe stenosis were recruited, and the $2 \mathrm{D}$ nature of the US technique used in this study. It is evident that 3D US could better and more thoroughly characterize plaque echogenicity and irregularities, though this technique is not widely available. The retrospective nature of this study is another limitation because the more vulnerable parts of the plaques may have already been dislodged causing a stroke. Even in this case, the underlying and remaining part of the symptomatic plaque might be expected to have a more irregular surface, and this feature was recorded in the present study. Other limitations of this study include the use of manual delineation of the plaque surface over still frames of carotid plaques, as opposed to the study by Kanber et $\mathrm{al}^{16,17}$ in which video clips and an automated method of plaque delineation were used. It has been previously found that variables such as the GSM may exhibit dynamic variations throughout the cardiac cycle, ${ }^{32}$ thus rendering their quantification more challenging. These along with the smaller number of patients recruited than in the study by Kanber et al may explain no statistical significance being achieved regarding the comparison of areas under the curve. Nevertheless and despite those shortcomings, this study yielded significant differences in the variables examined between symptomatic and asymptomatic plaques and confirmed the pattern of diagnostic accuracy previously reported. Future prospective studies should thus examine some form of multiparametric VI in prospective patient cohorts, validating these initial results in different vascular laboratories and investigating implications for patient management. It would also be very useful to compare symptomatic and asymptomatic patient populations with carotid disease.

On the basis of the currently available literature, factors not included in the present study also contribute to the vulnerability of the plaque. Namely, intraplaque neovascularization and the enhancement of the plaque on delayed-phase CEUS are 2 such factors, with vulnerable plaques having a greater degree of neovascularization. ${ }^{3}$ Similarly, late-phase enhancement on CEUS 6 minutes after the administration of microbubbles has been found to be greater in symptomatic plaques, due to the higher degree of inflammation found within these plaques. ${ }^{33}$ Studies have shown that symptomatic carotid plaques exhibit diffuse microbubble uptake at their base, something not happening in asymptomatic plaques. The areas showing enhancement on CEUS corresponded to areas with increased numbers of microvessels as demonstrated on immunohistologic staining of endarterectomy specimens. ${ }^{34}$

In a study enrolling 104 patients, $80 \%$ of symptomatic patients had a plaque showing enhancement on CEUS, while only $30 \%$ of asymptomatic patients had such a plaque, with this difference being significant. On quantitative analysis, the enhanced plaque intensity was significantly higher in symptomatic plaques. ${ }^{35}$ Even when subjectively quantifying plaque enhancement in 2 or 4 levels of classification, this classification significantly correlated with histologically detected neovascularization, intraplaque hemorrhage, and macrophage-rich areas. ${ }^{36}$ The presence of neovascularization in patients with symptomatic carotid plaques as detected with CEUS was also significantly associated with the occurrence of microembolic signals in the middle cerebral artery detected by transcranial US. The latter is considered a predictor of future risk of stroke in patients with carotid stenosis. ${ }^{37}$ Regarding plaque ulceration, the literature shows that it is an essential feature of plaque vulnerability, being associated with the occurrence of stroke. ${ }^{14}$ This entity can be studied ultrasonographically with both the conventional color Doppler technique and CEUS, though improved sensitivity has been found for the latter technique. ${ }^{14,38}$ Further investigation of these factors, especially in the setting of a multiparametric index, is important and could have implications for patient management on the grounds of treating plaques deemed more vulnerable with higher priority. Consequently, future studies should also evaluate multiparametric indexes incorporating even more features of vulnerability.

\section{CONCLUSIONS}

This study concluded that symptomatic plaques exhibit a significantly higher degree of stenosis, lower values of the GSM, and higher values of the Kanber vulnerability index, thus confirming previous findings and also further investigating the potential use of CEUS for plaque surface delineation. It is thus evident that the use of multiparametric vulnerability indices is advantageous and should be encouraged and further studied for the detection of symptomatic plaques, even with the use of conventional flowvisualization techniques such as color or power Doppler and not necessarily CEUS. Nevertheless, the examination of the SII with CEUS could be readily incorporated in a CEUS examination protocol performed for intraplaque neovascularization, another well-established feature of vulnerability, to maximize the information provided regarding the vulnerability of plaque.

\section{ACKNOWLEDGMENTS}

We thank Pagkalidou Eirini, MSc, Department of Hygiene and Epidemiology, School of Medicine, Aristotle University of Thessaloniki, Thessaloniki, Greece, for assisting us with statistical analysis performed in this study.

Disclosures: Vasileios Rafailidis_RELATED: Grant: Alexander S. Onassis Public Benefit Foundation in Greece, Comments: scholarship for PhD studies. Chrysostomos Xerras-UNRELATED: Employment: AHEPA University Hospital, Comments: resident in the First Department of Neurology. Enrico Grisan-UNRELATED: Grants/ Grants Pending: Italian Ministry. ${ }^{*}$ Paul S. Sidhu—RELATED: Support for Travel to Meetings for the Study or Other Purposes: Bracco S.p.A, Samsung, Comments: travel and lecture fees; UNRELATED: Consultancy: ITREAS, Comments: consulting for the assessment of venography images in clinical trials; Payment for Lectures Including Service on Speakers Bureaus: Philips Healthcare, Hiatchi, Siemens, Comments: lecture fees; Royalties: Taylor and Francis, owner of Hodder Inc, Thieme AG, Comments: book royalties. *Money paid to the institution.

\section{REFERENCES}

1. Aboyans V, Ricco JB, Bartelink MEL, et al; ESC Scientific Document Group. 2017 ESC Guidelines on the Diagnosis and Treatment of Peripheral Arterial Diseases, in collaboration with the European Society for Vascular Surgery (ESVS): document covering athero- 
sclerotic disease of extracranial carotid and vertebral, mesenteric, renal, upper and lower extremity arteries-Endorsed by the European Stroke Organization (ESO) The Task Force for the Diagnosis and Treatment of Peripheral Arterial Diseases of the European Society of Cardiology (ESC) and of the European Society for Vascular Surgery (ESVS). Eur Heart J 2018;39:763-816 CrossRef Medline

2. Saba L, Yuan C, Hatsukami TS, et al; Vessel Wall Imaging Study Group of the American Society of Neuroradiology. Carotid artery wall imaging: perspective and guidelines from the ASNR Vessel Wall Imaging Study Group and Expert Consensus Recommendations of the American Society of Neuroradiology. AJNR Am J Neuroradiol 2018;39:E9-31 CrossRef Medline

3. Saba L, Anzidei M, Marincola BC, et al. Imaging of the carotid artery vulnerable plaque. Cardiovasc Intervent Radiol 2014;37:572-85 CrossRef Medline

4. MRC European Carotid Surgery Trial: interim results for symptomatic patients with severe $(70-99 \%)$ or with mild $(0-29 \%)$ carotid stenosis. European Carotid Surgery Trialists' Collaborative Group. Lancet 1991;337:1235-43 CrossRef Medline

5. Barnett HJ, Taylor DW, Haynes RB, et al; North American Symptomatic Carotid Endarterectomy Trial Collaborators. Beneficial effect of carotid endarterectomy in symptomatic patients with high-grade carotid stenosis. N Engl J Med 1991;325:445-53 CrossRef Medline

6. Tahmasebpour HR, Buckley AR, Cooperberg PL, et al. Sonographic examination of the carotid arteries. Radiographics 2005;25:1561-75 CrossRef Medline

7. Pedro LM, Pedro MM, Goncalves I, et al. Computer-assisted carotid plaque analysis: characteristics of plaques associated with cerebrovascular symptoms and cerebral infarction. Eur J Vasc Endovasc Surg 2000;19:118-23 CrossRef Medline

8. Biasi GM, Sampaolo A, Mingazzini P, et al. Computer analysis of ultrasonic plaque echolucency in identifying high risk carotid bifurcation lesions. Eur J Vasc Endovasc Surg 1999;17:476-79 CrossRef Medline

9. Elatrozy T, Nicolaides A, Tegos T, et al. The objective characterisation of ultrasonic carotid plaque features. Eur J Vasc Endovasc Surg 1998;16:223-30 CrossRef Medline

10. Grønholdt ML, Nordestgaard BG, Schroeder TV, et al. Ultrasonic echolucent carotid plaques predict future strokes. Circulation 2001; 104:68-73 CrossRef Medline

11. Tegos TJ, Stavropoulos P, Sabetai MM, et al. Determinants of carotid plaque instability: echoicity versus heterogeneity. Eur J Vasc Endovasc Surg 2001;22:22-30 CrossRef Medline

12. Salem MK, Sayers RD, Bown MJ, et al. Patients with recurrent ischaemic events from carotid artery disease have a large lipid core and low GSM. Eur J Vasc Endovasc Surg 2012;43:147-53 CrossRef Medline

13. Ruiz-Ares G, Fuentes B, Martinez-Sanchez P, et al. Utility of the assessment of echogenicity in the identification of symptomatic carotid artery atheroma plaques in ischemic stroke patients. Cerebrovasc Dis 2011;32:535-41 CrossRef Medline

14. Rafailidis V, Chryssogonidis I, Tegos T, et al. Imaging of the ulcerated carotid atherosclerotic plaque: a review of the literature. Insights Imaging 2017;8:213-25 CrossRef Medline

15. Tegos TJ, Kalomiris KJ, Sabetai MM, et al. Significance of sonographic tissue and surface characteristics of carotid plaques. AJNR Am J Neuroradiol 2001;22:1605-12 Medline

16. Kanber B, Hartshorne TC, Horsfield MA, et al. Quantitative assessment of carotid plaque surface irregularities and correlation to cerebrovascular symptoms. Cardiovasc Ultrasound 2013;11:38 CrossRef Medline

17. Kanber B, Hartshorne TC, Horsfield MA, et al. A novel ultrasound-based carotid plaque risk index associated with the presence of cerebrovascular symptoms. Ultraschall Med 2015;36: 480-86 CrossRef Medline

18. Rafailidis V, Charitanti A, Tegos T, et al. Contrast-enhanced ultrasound of the carotid system: a review of the current literature. $J$ Ultrasound 2017;20:97-109 CrossRef Medline

19. Piscaglia F, Nolsøe C, Dietrich CF, et al. The EFSUMB Guidelines and Recommendations on the Clinical Practice of Contrast Enhanced Ultrasound (CEUS): update 2011 on non-hepatic applications. Ultraschall Med 2012;33:33-59 CrossRef Medline

20. Sidhu PS, Cantisani V, Dietrich CF, et al. The EFSUMB Guidelines and Recommendations for the Clinical Practice of Contrast-Enhanced Ultrasound (CEUS) in Non-Hepatic Applications: Update 2017 (Long Version). Ultraschall Med 2018;39:e2-44 CrossRef Medline

21. Grant EG, Benson CB, Moneta GL, et al. Carotid artery stenosis: gray-scale and Doppler US diagnosis-Society of Radiologists in Ultrasound Consensus Conference. Radiology 2003;229:340-46 CrossRef Medline

22. Sabetai MM, Tegos TJ, Nicolaides AN, et al. Reproducibility of computer-quantified carotid plaque echogenicity: can we overcome the subjectivity? Stroke 2000;31:2189-96 CrossRef Medline

23. Prati P, Tosetto A, Casaroli M, et al. Carotid plaque morphology improves stroke risk prediction: usefulness of a new ultrasonographic score. Cerebrovasc Dis 2011;31:300-04 CrossRef Medline

24. Momjian-Mayor I, Kuzmanovic I, Momjian S, et al. Accuracy of a novel risk index combining degree of stenosis of the carotid artery and plaque surface echogenicity. Stroke 2012;43:1260-65 CrossRef Medline

25. Pedro LM, Fernandes e Fernandes J, Pedro MM, et al. Ultrasonographic risk score of carotid plaques. Eur J Vasc Endovasc Surg 2002; 24:492-98 CrossRef Medline

26. Afonso D, Seabra J, Suri JS, et al. A CAD system for atherosclerotic plaque assessment. Conf Proc IEEE Eng Med Biol Soc 2012;2012: 1008-11 CrossRef Medline

27. Nicolaides AN, Kakkos SK, Kyriacou E, et al; Asymptomatic Carotid Stenosis and Risk of Stroke (ACSRS) Study Group. Asymptomatic internal carotid artery stenosis and cerebrovascular risk stratification. J Vasc Surg 2010;52:1486-96 e1-5 CrossRef Medline

28. Kono Y, Pinnell SP, Sirlin CB, et al. Carotid arteries: contrast-enhanced US angiography-preliminary clinical experience. Radiology 2004;230:561-68 CrossRef Medline

29. Schinkel AF, Kaspar M, Staub D. Contrast-enhanced ultrasound: clinical applications in patients with atherosclerosis. Int J Cardiovasc Imaging 2016;32:35-48 CrossRef Medline

30. Staub D, Partovi S, Imfeld S, et al. Novel applications of contrastenhanced ultrasound imaging in vascular medicine. Vasa 2013;42: 17-31 CrossRef Medline

31. Eyding J, Geier B, Staub D. Current strategies and possible perspectives of ultrasonic risk stratification of ischemic stroke in internal carotid artery disease. Ultraschall Med 2011;32:267-73 CrossRef Medline

32. Kanber B, Hartshorne TC, Horsfield MA, et al. Dynamic variations in the ultrasound greyscale median of carotid artery plaques. Cardiovasc Ultrasound 2013;11:21 CrossRef Medline

33. Owen DR, Shalhoub J, Miller S, et al. Inflammation within carotid atherosclerotic plaque: assessment with late-phase contrast-enhanced US. Radiology 2010;255:638-44 CrossRef Medline

34. Giannoni MF, Vicenzini E, Citone M, et al. Contrast carotid ultrasound for the detection of unstable plaques with neoangiogenesis: a pilot study. Eur J Vasc Endovasc Surg 2009;37:722-27 CrossRef Medline

35. Xiong L, Deng YB, Zhu Y, et al. Correlation of carotid plaque neovascularization detected by using contrast-enhanced US with clinical symptoms. Radiology 2009;251:583-89 CrossRef Medline

36. Schmidt C, Fischer T, Rückert RI, et al. Identification of neovascularization by contrast-enhanced ultrasound to detect unstable carotid stenosis. PLoS One 2017;12:e0175331 CrossRef Medline

37. Ritter MA, Theismann K, Schmiedel M, et al. Vascularization of carotid plaque in recently symptomatic patients is associated with the occurrence of transcranial microembolic signals. Eur J Neurol 2013; 20:1218-21 CrossRef Medline

38. Rafailidis V, Chryssogonidis I, Xerras C, et al. A comparative study of color Doppler imaging and contrast-enhanced ultrasound for the detection of ulceration in patients with carotid atherosclerotic disease. Eur Radiol 2019;29:2137-45 CrossRef Medline 